\title{
Femtosecond Dynamics of the Exciton Self-Trapping Process in a Quasi-One-Dimensional Halogen-Bridged Platinum Complex
}

\author{
S. Tomimoto ${ }^{1}$ H. Nansei, ${ }^{1}$ S. Saito,${ }^{1}$ T. Suemoto,${ }^{1}$ J. Takeda,${ }^{2}$ and S. Kurita ${ }^{2}$ \\ ${ }^{1}$ Institute for Solid State Physics, University of Tokyo, Minato-ku, Tokyo 106-8666, Japan \\ ${ }^{2}$ Department of Physics, Faculty of Engineering, Yokohama National University, Yokohama 240-8501, Japan
}

(Received 29 December 1997)

Self-trapped-exciton (STE) luminescence of quasi-one-dimensional halogen-bridged platinum complex $\left[\mathrm{Pt}(\mathrm{en})_{2}\right]\left[\mathrm{Pt}(\mathrm{en})_{2} \mathrm{Cl}_{2}\right]\left(\mathrm{ClO}_{4}\right)_{4}$ is investigated by femtosecond time-resolved luminescence spectroscopy. In the high energy tail of the luminescence band, a fast rise and decay are seen, whereas around the luminescence peak energy a slow rise of about $500 \mathrm{fs}$ is observed. This rise becomes faster at the low energy side of the band. These results are consistently explained by the cooling of prethermalized STEs, following the rapid self-trapping which is estimated to occur within $140 \mathrm{fs}$. [S0031-9007(98)06543-0]

PACS numbers: 71.35.Aa, 78.47.+p, 78.55.Hx

Charge carriers and excitons photogenerated in a crystal with strong electron-phonon interaction are known to relax to self-trapped states causing local lattice deformation. The dynamical aspects of self-trapping have been investigated in many insulators by taking advantage of the ultrashort pulsewidths of recent lasers [1]. Most of the measurements have been done by the pump and probe transient absorption method. As an experimental method of studying exciton self-trapping, however, time-resolved luminescence spectroscopy is equally powerful.

The self-trapping process critically depends on the dimensionality of the system [1]. In a three-dimensional (3D) system, an adiabatic potential barrier exists between free and self-trapped states. Thus the rise time of selftrapped exciton (STE) luminescence usually represents the time needed for excitons to overcome the potential barrier $[2,3]$. In a one-dimensional (1D) system, on the contrary, it is believed that there is no potential barrier to self-trapping, and the time scale of the barrier-free relaxation is expected to be extremely short [4]. Consequently, the rise time of STE luminescence has been well resolved in 3D crystals, such as alkali halides [2] and pyrene [3], but has not been adequately measured in 1D systems [5]. For this reason, the observation of the ultrafast behavior of STE luminescence in 1D systems is considered as very interesting subject.

Halogen-bridged mixed-valence platinum complex $\left[\mathrm{Pt}(\mathrm{en})_{2}\right]\left[\mathrm{Pt}(\mathrm{en})_{2} \mathrm{Cl}_{2}\right]\left(\mathrm{ClO}_{4}\right)_{4}$ (en = ethylenediamine) is a quasi-1D crystal consisting of linear chains of alternating platinum and chlorine ions. The structure of the linear chain can be described as the sequence $\cdots-\mathrm{Pt}^{2+}-$ $\mathrm{Cl}^{-}-\mathrm{Pt}^{4+}-\mathrm{Cl}^{-}-\cdots$, where the chlorine ions are located closer to $\mathrm{Pt}^{4+}$ than to $\mathrm{Pt}^{2+}$. This structure can be understood as a 1D charge-density wave (CDW) caused by the displacement of chlorine ions from the central position of the neighboring platinum ions [6]. The crystal exhibits an intense chain-axis-polarized absorption with a low energy edge near $2.4 \mathrm{eV}$ [6]. This absorption has been assigned to a charge-transfer (CT) optical excitation of an electron from $\mathrm{Pt}^{2+}$ to $\mathrm{Pt}^{4+}$ which gives a pair of $\mathrm{Pt}^{3+}$. When the crystal is excited above the absorption edge, an intense broad luminescence band due to STE appears around $1.2 \mathrm{eV}$ [7]. By picosecond time-resolved luminescence measurements, it has been revealed that this luminescence shows single exponential decay with a lifetime less than $100 \mathrm{ps}$ at room temperature and that an additional fast decay component appears at the high energy tail of the band $[8,9]$. As for the lifetime of the fast decay component, it is recently reported to be around 1 ps [10].

In this Letter, we report the experimental results of femtosecond time-resolved measurement of the luminescence band. We have investigated the time evolution of the luminescence over a wide emission energy range including the peak energy. From the dependence of the luminescence rise time on emission photon energy, we will discuss the dynamical aspects of excitons during equilibration to the STE potential minimum. This is the first successful determination of the STE luminescence rise time in the subpicosecond time regime as far as the authors know.

The experiment has been done by means of the luminescence up-conversion technique. The single crystal of $\left[\mathrm{Pt}(\mathrm{en})_{2}\right]\left[\mathrm{Pt}(\mathrm{en})_{2} \mathrm{Cl}_{2}\right]\left(\mathrm{ClO}_{4}\right)_{4}$ is prepared by the procedure described elsewhere [7]. The sample is excited at $3.2 \mathrm{eV}$ by the second harmonic of a mode-locked Ti:sapphire laser with a repetition rate of $90 \mathrm{MHz}$. This excitation light is polarized parallel to the chain axis of the crystal, and the intensity is kept at $1.2 \mathrm{~mW}\left(3.8 \mathrm{~W} / \mathrm{cm}^{2}\right)$. The luminescence from the sample at room temperature is collected by paraboloidal mirrors and is mixed with variably delayed pulses at the fundamental frequency of the laser in a nonlinear crystal of $\mathrm{LiIO}_{3}$. The sum frequency output is focused into a double monochromator and is detected by a photomultiplier tube and a photon counter. The overall time resolution of the system is about $180 \mathrm{fs}$, which is determined by a cross-correlation measurement of the scattered excitation light and the delayed laser pulses. 
Figure 1 shows the time dependence of luminescence intensity measured at different photon energies. The photon energy range observed in this study is indicated by arrows in Fig. 2 on the time-integrated luminescence spectrum. As Fig. 1 shows, the STE luminescence decays slowly with a lifetime longer than $20 \mathrm{ps}$. In addition to this slow decay component, a fast component which decays within $1 \mathrm{ps}$ appears at the high energy tail of the band (above $1.8 \mathrm{eV}$ ). The relative intensity of the fast component increases with increasing emission photon energy. The function

$$
I(t)=I_{0}\left(1-e^{-t / \tau_{r}}\right) e^{-t / \tau_{d}}
$$

is fitted to the data for the photon energy below $1.4 \mathrm{eV}$. The fitting curves shown as broken ones in Fig. 1 are obtained by the convolution of Eq. (1) with the system response function. Around the luminescence peak energy (from 1.0 to $1.4 \mathrm{eV}$ ), the time evolution of luminescence is well expressed as single exponential decay. The observed photon energy dependence of the fitting parameters $\tau_{r}, \tau_{d}$ is shown in Fig. 2(a). The lifetime of the slow decay component $\tau_{d}$ is slightly scattered around $25 \mathrm{ps.} \mathrm{However,}$ no obvious dependence on the photon energy is observed. Based on its temperature dependence, this slow decay component has been assigned to the thermally activated nonradiative annihilation of STEs by Wada et al. [9]. Their value of $\tau_{d}$ (about $70 \mathrm{ps}$ around $270 \mathrm{~K}$ ), however, is rather longer than what we measured. We attribute this differ-

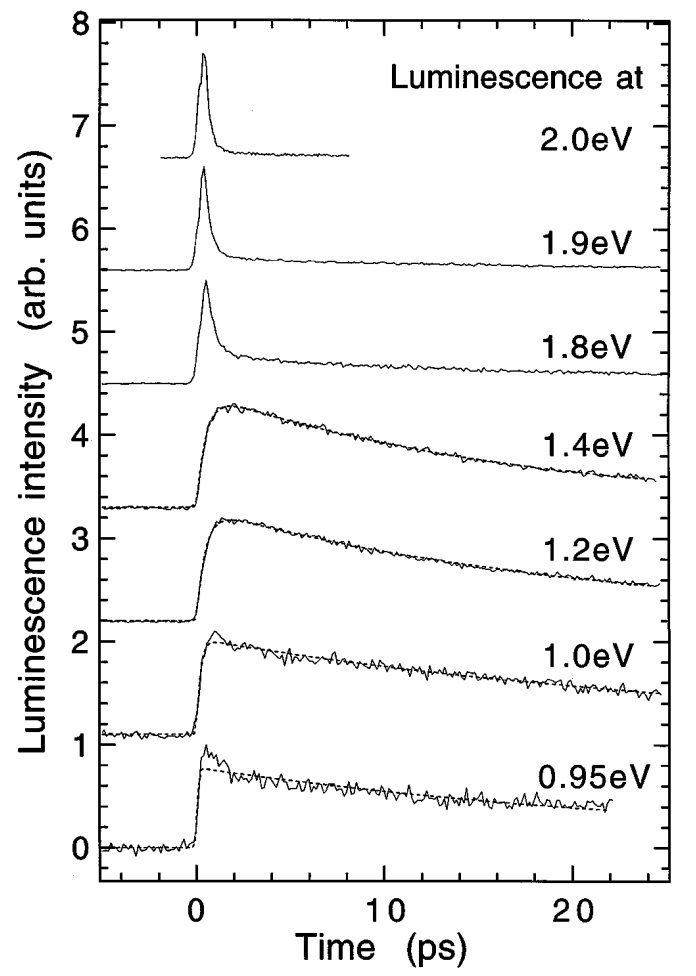

FIG. 1. Time evolution of luminescence measured at different photon energies. Solid curves are experimental data. Broken curves shown below $1.4 \mathrm{eV}$ are the fitting curves based on Eq. (1). Intensities are normalized and base lines are shifted for clarity. ence to the large difference in the observed time range. In the low energy tail of the band (below $1.0 \mathrm{eV}$ ), the time evolution of luminescence is no longer expressed as single exponential decay. As can be seen in Fig. 1, a fast decay component appears again. But the relative intensity of this component is small in the observed energy range.

The time evolution of luminescence from -2 to $5 \mathrm{ps}$ is shown in Fig. 3. The lifetime of the fast decay component observed in the high energy tail of the band (from 1.8 to $2.1 \mathrm{eV}$ ) is several hundred femtoseconds. This component decays faster in higher energies. In contrast to the fast rise and decay seen in the high energy tail, a slow rise is observed around the luminescence peak energy (about $1.2 \mathrm{eV}$ ). This rise becomes faster in the low energy side of the band. As shown in Fig. 2(a), the luminescence rise time $\tau_{r}$ is about 500 fs around the peak energy and becomes much shorter in the low energy side. The upper limit of the rise time at $0.95 \mathrm{eV}$ is determined to be $140 \mathrm{fs}$ from a fitting of the function Eq. (1) to the observed time evolution. At $0.95 \mathrm{eV}$, the fast rise is followed by a slight fast decrease within 2 ps.

The origin of the fast decay component seems to be different from that of the 1.75 ps component observed in transient absorption [11], because this component was observed only at $3.1 \mathrm{eV}$ excitation, while the fast component in luminescence is observed also at the excitation around the CT absorption edge [9]. The component in transient absorption could be attributed to different species to which our luminescence measurement is insensitive.

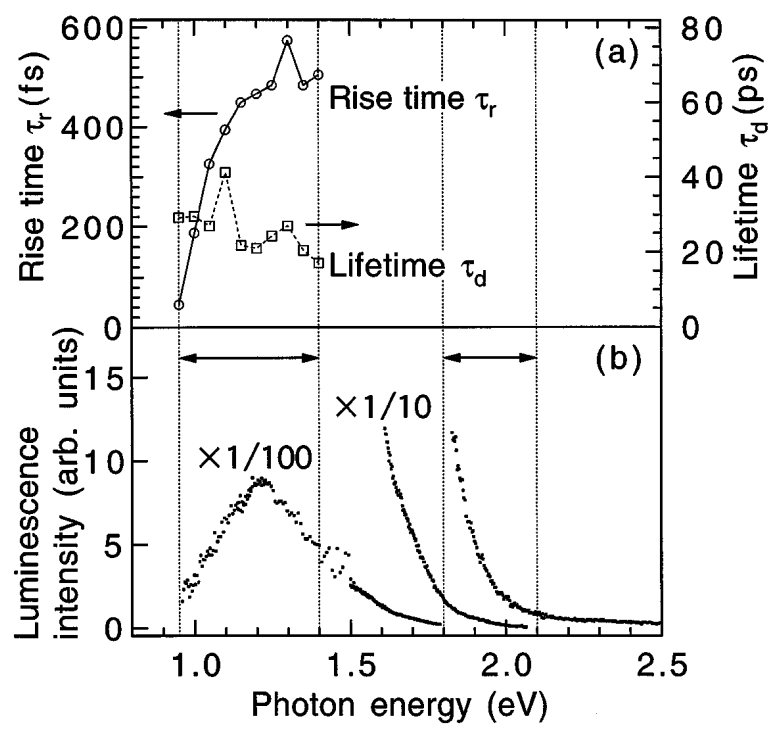

FIG. 2. (a) Emission photon energy dependence of the luminescence rise time $\tau_{r}$ (circles) and the lifetime of the slow decay component $\tau_{d}$ (squares). (b) Time-integrated luminescence spectrum under the same excitation as the time-resolved measurement. The time evolution of luminescence is observed at the energy range indicated by arrows (from 0.95 to $1.4 \mathrm{eV}$ and from 1.8 to $2.1 \mathrm{eV}$ ). It is impossible to observe it from 1.4 to $1.8 \mathrm{eV}$ because of the difficulty of eliminating the stray light due to the second harmonic of the gating pulses. 


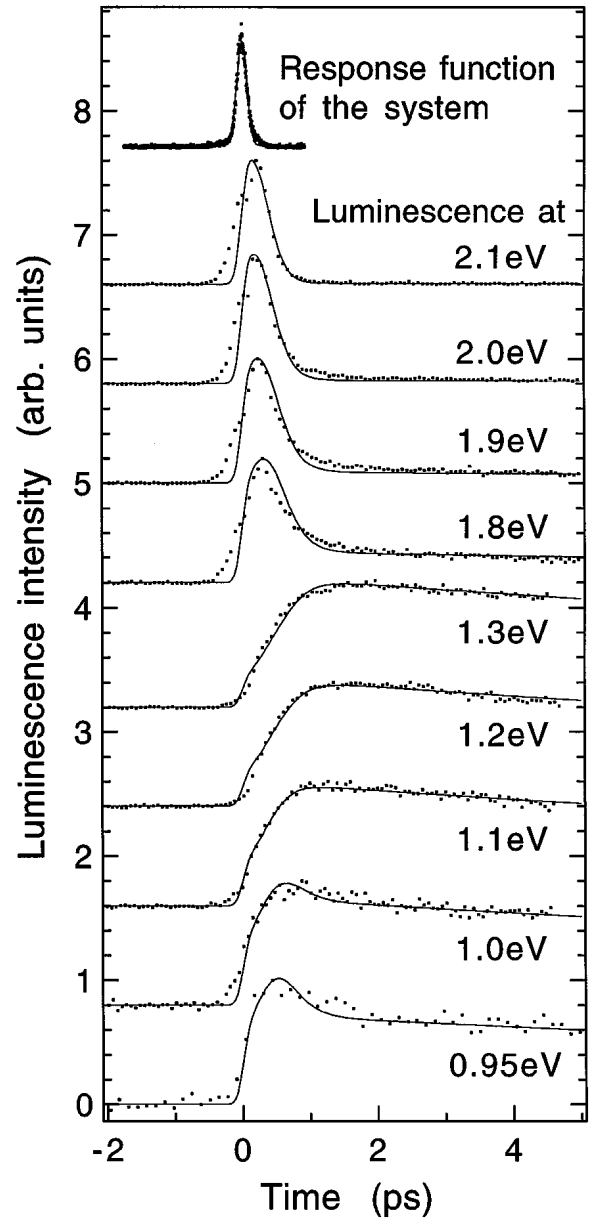

FIG. 3. Time evolution of luminescence from -2 to $5 \mathrm{ps}$. Dots are experimental data. Solid curves show the results of the calculation based on Eq. (2). Intensities are normalized. The zero time delay for each photon energy has been determined by fitting the solid curves to the experimental data. The response function of the system is shown at the top of the figure.

In order to understand the above-mentioned complicated behavior qualitatively, we consider the self-trapping dynamics using the adiabatic potential energy curves illustrated in Fig. 4(a). The abscissa $Q$ is the lattice distortion which represents the positions of $\mathrm{Cl}^{-}$relative to the platinum ions. Here $Q_{0}$ is the configuration of the CDW ground state, where the chlorine ions are situated closer to $\mathrm{Pt}^{4+}$ than to $\mathrm{Pt}^{2+}$. The lowest excited state is a CT exciton consisting of a pair of $\mathrm{Pt}^{3+}$. In an ideal 1D electronphonon system, there is no potential barrier between the free $(\alpha)$ and self-trapped $(\beta)$ states. Accordingly the photogenerated $\mathrm{CT}$ excitons will readily relax to the localized self-trapped state. At the STE potential minimum $(\beta)$, $\mathrm{Cl}^{-}$is expected to be displaced to the point close to the central position of neighboring $\mathrm{Pt}^{3+}$ ions.

Three processes have to be examined as candidates for the process which determines the luminescence rise time: (1) the localization of free CT excitons, (2) the movement of a wave packet from the undistorted state $(\alpha)$ to the po-

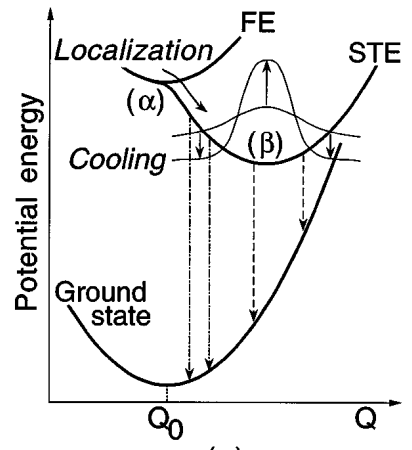

(a)

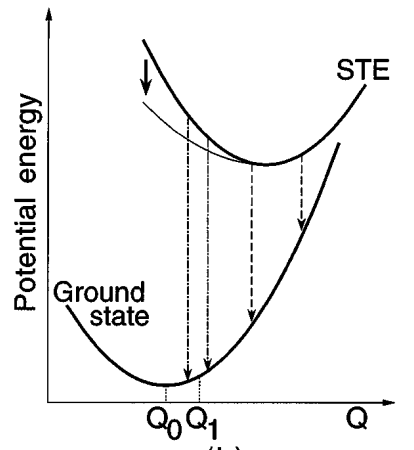

(b)
FIG. 4. (a) Adiabatic potential energy curves in a configuration space. The distribution change in the process of "cooling" is schematically depicted. The transition in the regions between dashed lines and between dot-dashed lines will correspond to the luminescence observed in our experiment. (b) A possible anharmonic potential of the excited state is depicted as a thin curve.

tential minimum $(\beta)$, and (3) the thermalization or cooling of "hot" STEs. As the luminescence at $0.95 \mathrm{eV}$ rises within $140 \mathrm{fs}$, the exciton localization (1) is considered to occur within this time constant. Therefore the much slower rise of about $500 \mathrm{fs}$ around the peak energy should be assigned to the process (2) or (3). In the picture of the wave packet movement (2), the fast decay component observed in the high energy tail of the band can be attributed to radiative recombination during this movement. This picture is, however, inappropriate, considering that the period of the wave packet oscillation in the excited state is expected to be about $100 \mathrm{fs}$ according to the estimation from the period of the breathing vibration of $\mathrm{Cl}^{-}$around $\mathrm{Pt}^{4+}$ along the chain in the CDW ground state [7]. This period is far shorter than the $500 \mathrm{fs}$ luminescence rise time. Furthermore, in this picture, the luminescence in the low energy side of the band should rise as slowly as, or more slowly than, that around the peak energy. In our experiment, however, the luminescence rises faster at the low energy side. As we will discuss below, we interpret the $500 \mathrm{fs}$ luminescence rise time as the time for the cooling of hot STEs (3).

Just after the localization of CT excitons, the STEs will have large excess vibrational energy. In a certain time after the localization, the excess energy will be dissipated, and the hot STEs will be cooled to thermalize around the potential minimum $(\beta)$ at room temperature. We have schematically depicted the distribution change in Fig. 4(a). If we assume that the time evolution of luminescence reflects this cooling or equilibration of STEs, the luminescence around the peak energy will take longer to rise to the maximum than that at a little higher or lower energy. The luminescence in the high or low energy tail of the band will decrease during the equilibration. In this picture, therefore, the luminescence in the low energy side could rise faster than that around the peak energy. The fast 
component observed in the high energy tail of the band and the slight fast decrease observed in the low energy tail can be attributed to the luminescence of hot STEs during the equilibration.

In order to illustrate the cooling picture, we have made the following calculation and plotted calculated luminescence curves in Fig. 3. Although the distribution of STEs in the potential during cooling will be really a nonequilibrium one, we have tentatively assumed it to be the Boltzmann distribution at any time for simplicity. From this assumption, considering the lattice vibration classically, the time-resolved luminescence spectrum is always expressed as a Gaussian band shape. The luminescence intensity at time $t$ and photon energy $E$ is expressed as follows:

$$
f(t, E)=f_{0} T(t)^{-1 / 2} \exp \left\{-\left(E-E_{0}\right)^{2} / w T(t)\right\} .
$$

By assuming that the "temperature" of STEs decays exponentially to the room temperature $T_{0}(=300 \mathrm{~K})$, we have expressed the cooling

$$
T(t)=T_{0}+T_{1} e^{-t / \tau} .
$$

The initial temperature $T_{0}+T_{1}(=7000 \mathrm{~K})$ is determined from the lattice relaxation energy, i.e., half of the energy separation between the CT absorption energy and the luminescence peak energy. We have determined the other parameters as follows: the luminescence peak energy $E_{0}=1.3 \mathrm{eV}$, the band width parameter $w=3 \times$ $10^{-4} \mathrm{eV}^{2} / \mathrm{K}$, and the time constant of cooling or equilibration $\tau=0.21$ ps. The solid curves shown in Fig. 3 have been obtained from the convolution of Eq. (2) with the system response function and from the assumption that the STEs decay exponentially with the lifetime of $25 \mathrm{ps}$.

The calculated curves are in good agreement with the experimental data. These curves reproduce the following important features of the experimental results: the fast rise and decay in the high energy tail of the band (above $1.8 \mathrm{eV}$ ), the faster rise at lower energy in the low energy side of the band, and the appearance of the slight fast decrease in the low energy tail of the band (below $1.0 \mathrm{eV}$ ). The result of the calculation leads us to the conclusion that the rise time of about 500 fs around the peak energy represents the time for the cooling of hot STEs.

The parameters $E_{0}$ and $w$ used in the calculation give a luminescence spectrum at $300 \mathrm{~K}$ rather deviated from the spectrum shown in Fig. 2(b). If we assumed $E_{0}=$ $1.21 \mathrm{eV}$ and $w=2.3 \times 10^{-4} \mathrm{eV}^{2} / \mathrm{K}$, we could obtain the best fitting to the spectrum around the peak. However, if these values were used, the calculated relative intensity of the slow decay component to the fast component in the high energy tail (above $1.8 \mathrm{eV}$ ) would be significantly smaller than the observed one. The rather strong relative intensity of the slow decay component observed in the high energy tail could be qualitatively explained considering anharmonicity in the excited-state potential. A possible anharmonic potential is depicted in Fig. 4(b) as a thin curve. It will increase the population of STEs around $Q_{1}$ in thermal equilibrium, and will make the relative intensity of the slow decay component in the high energy tail stronger than that in the case of a completely harmonic potential.

The time-integrated luminescence spectrum shown in Fig. 2(b) has fairly strong intensity in the high energy tail. This cannot be ascribed to the fast decay component, i.e., the hot luminescence, because the time-integrated intensity of the fast component relative to the slow decay component is estimated to be only $1 / 8$ at $1.8 \mathrm{eV}$ and $1 / 4$ at $1.9 \mathrm{eV}$ from the experimental data shown in Fig. 3. The observed band shape which has fairly strong intensity in the high energy tail could be qualitatively explained by the same anharmonic potential as the one illustrated in Fig. 4(b).

In conclusion, we observed the femtosecond timeresolved luminescence in a wide energy region which included the luminescence peak energy for the STE in the 1D system, $\left[\mathrm{Pt}(\mathrm{en})_{2}\right]\left[\mathrm{Pt}(\mathrm{en})_{2} \mathrm{Cl}_{2}\right]\left(\mathrm{ClO}_{4}\right)_{4}$. The experimental results and the calculation lead us to the conclusion that the slow rise of about $500 \mathrm{fs}$ around the luminescence peak energy does not represent the time for the movement of a wave packet from the free state to the self-trapped state, but gives the time of cooling or equilibration of STEs around the potential minimum. The localization of free CT excitons is considered to occur within $140 \mathrm{fs}$ from the estimation of the upper limit of the rise time at $0.95 \mathrm{eV}$.

[1] K.S. Song and R.T. Williams, Self-Trapped Excitons, Springer Series in Solid State Sciences Vol. 105 (Springer-Verlag, Berlin, 1993).

[2] Y. Unuma, Y. Masumoto, S. Shionoya, and H. Nishimura, J. Phys. Soc. Jpn. 52, 4277 (1983).

[3] A. Matsui, K. Mizuno, N. Tamai, and I. Yamazaki, Chem. Phys. 113, 111 (1987).

[4] M. Yoshizawa, K. Nishiyama, M. Fujihira, and T. Kobayashi, Chem. Phys. Lett. 207, 461 (1993).

[5] R. Kersting et al., Phys. Rev. Lett. 70, 3820 (1993).

[6] Y. Wada, T. Mitani, M. Yamashita, and T. Koda, J. Phys. Soc. Jpn. 54, 3143 (1985).

[7] M. Tanaka et al., Chem. Phys. 96, 343 (1985).

[8] H. Tanino, W. W. Rühle, and K. Takahashi, Phys. Rev. B 38, 12716 (1988).

[9] Y. Wada et al., Phys. Rev. B 52, 8276 (1995).

[10] T. Kobayashi, T. Sekikawa, and M. Yamashita, Chem. Lett. 10, 1029 (1997).

[11] G. S. Kanner et al., Phys. Rev. B 56, 2501 (1997). 